\title{
Diagnostic value of lung ultrasound for neonatal respiratory distress syndrome: a meta-analysis and systematic review
}

\author{
Hai-Ran Ma ${ }^{1,2}$, Jing Liu², Wen-Kang Yan' \\ ${ }^{1}$ Department of Neonatology and Neonatal Intensive Care Unit, Huizhou Municipal Central Hospital, Huizhou \\ Guangdong, ${ }^{2}$ Department of Neonatology and Neonatal Intensive Care Unit, Beijing Chaoyang District Maternal and \\ Child Healthcare Hospital, Beijing and National Neonatal Lung Ultrasound Training Base, Beijing, China
}

\begin{abstract}
Aim: Neonatal respiratory distress syndrome (NRDS) is one of the most common and severe diseases in neonatal intensive care units worldwide. Increasing evidence suggests that lung ultrasound (LUS) may be a reliable diagnostic tool for neonatal respiratory distress syndrome. The aim of study was to evaluate the diagnostic accuracy of LUS for NRDS with a systematic review and meta-analysis. Material and methods: We searched for articles in EMBASE, PubMed and Cochrane Central from inception until 17 August 2019. The selected studies were diagnostic accuracy studies that reported the utility of LUS in the diagnosis of NRDS. Two researchers independently extracted data and assessed quality using the QUADAS-2 tool. Then, we created a bivariate mixed effects model to calculate the sensitivity and specificity of LUS in diagnosing NRDS. A summary receiver operator characteristic (SROC) curve was constructed to summarize the performance characteristics of LUS. Results: Nine studies involving 703 infants were included in the review. LUS had a pooled sensitivity of 0.99 (CI: 0.92-1.00) and a specificity of 0.95 (CI: 0.87-0.98). The areas under the curve for LUS was 0.99 (0.98-1.0). Meta-regression revealed that LUS had a significant diagnostic accuracy for NRDS. Conclusion: LUS is a promising method that is easily carried out, inexpensive, nonionizing and repeatable and can be performed at the bedside. Current evidence supports LUS as a useful imaging alternative for the diagnosis of NRDS.
\end{abstract}

Keywords: ultrasonography; respiratory distress syndrome; newborn

\section{Introduction}

Neonatal respiratory distress syndrome (NRDS) usually occurs in preterm infants. This disorder is caused primarily by surfactant deficiency that leads to diffuse atelectasis and alveolar collapse shortly after birth. NRDS is one of the most important causes of morbidity and mortality in preterm infants. The clinical features of NRDS

Received 29.02.2020 Accepted 02.04.2020

Med Ultrason

2020, Vol. 22, No 3, 325-333

Corresponding author: Jing Liu

Department of Neonatology and Neonatal Intensive Care Unit, Beijing Chaoyang District Maternal and Child Healthcare Hospital

25 Huaweili street, Panjiayuan,

Chaoyang District, 100101 Beijing, China

Phone: 086-13301195869

E-mail: liujingbj@live.cn are characterized by tachypnoea, nasal flaring, expiratory grunting, chest retractions and cyanosis [1], and the risk for this syndrome increases with decreasing gestational age (GA). The incidence of NRDS was found to be $93 \%$ in extremely preterm infants (GA 28 weeks or below) [2] and $10.5 \%, 6 \%, 2.8 \%, 1 \%$ and $0.3 \%$ in infants born at $34,35,36,37$, and $\geq 38$ weeks gestation, respectively [3].

Tremendous efforts have been made to improve the morbidity and mortality of NRDS. There are well-established methods for the diagnosis and treatment of NRDS. Early diagnosis is important to optimize the treatment of infants with NRDS. Chest radiography (CXR) is currently a leading and extensive tool in establishing the diagnosis of NRDS [4,5], although currently, the appearance of "ground glass with air bronchograms" is rarely seen due to early surfactant therapy and early continuous positive airway pressure (CPAP) [5]. However, CXR has the risk of exposing the infant to ionizing radiation. The risk 
for the potential negative effects of CXR are significantly higher in younger children [6,7]. A 1-year-old child is 1015 times more at risk of developing carcinoma than an adult who receives the same dose of ionizing radiation [8].

Lung ultrasound (LUS), as a non-invasive routine technology, was used to diagnose neonatal lung diseases at the beginning of the last decade of the $20^{\text {th }}$ century $[9,10]$. LUS has recently been recommended as a diagnostic procedure in the diagnosis and monitoring of NRDS in clinical practice [5]. The advantages of an ultrasound examination over CXR, such as its non-ionizing, convenient and repeatable nature, in the diagnosis of NRDS in neonates were shown by a number of publications [9,11-15]. Two ultrasonography approaches (transthoracic and transabdominal) were used to detect NRDS and both had high sensitivity and relatively high specificity $[12,16]$. Therefore, LUS appears to be the ideal choice for a screening or diagnostic method in the assessment of NRDS in newborns. Nonetheless, LUS has a tendency to overdiagnose NRDS [17]. A comprehensive systematic review with a structured question is required to identify the diagnostic accuracy, methodological quality of evidence, limitations and clinical application of LUS for detecting NRDS in the contemporary era. The objective of this review is to calculate the pooled sensitivity and specificity of LUS in diagnosing NRDS and facilitate an understanding of the capabilities of LUS regarding the evaluation of NRDS.

\section{Material and method}

\section{Data sources and searches}

In this systematic review and meta-analysis, two researchers (J. L and HR. M) independently searched the PubMed, EMBASE and Cochrane Library databases for articles published in any language or country [18]. These general bibliographic databases were searched from inception to 17 August 2019. We believe this search strategy is sufficient for this study since searching Medline (part of PUBMED) leads to only minor differences in the estimates of diagnostic test accuracy [19]. Aside from the above search, the reference lists of the included studies were checked manually to identify as many relevant diagnostic test accuracy (DTA) studies as possible [20].

The search used Medical Subject Headings terms for neonates, ultrasound and NRDS. The Boolean operators (AND) and (OR) were used to minimize irrelevant literature and maximize the breadth of the search. The following search terms for ultrasonography were combined using the OR connector: "echotomography" OR "echography" OR "sonography" OR "medical sonogra- phy" OR "ultrasonography" OR "ultrasound" OR "ultrasonic tomography" OR "computer echotomography". Similarly, the search terms for NRDS were defined using the following syntax: "Neonatal Respiratory Distress Syndrome" OR "Respiratory Distress Syndrome" OR "Infantile Respiratory Distress Syndrome" OR "Hyaline Membrane Disease" OR "Hyaline Membrane Diseases" OR "Disease, Hyaline Membrane". The search results of ultrasonography and neonatal respiratory distress syndrome were combined using the AND connector. Discrepancies between researchers were resolved through a consensus with a third reviewer (WK. Y).

\section{Study selection}

All articles identified as potentially eligible by either reviewer were reviewed as abstracts or full-text articles. Eligible studies met the following inclusion criteria: 1) cohort or case-control studies used LUS as a diagnostic tool for neonatal respiratory distress syndrome; 2) NRDS was diagnosed using a combination of clinical signs and symptoms (tachypnoea, nasal flaring, chest retractions and grunting), CXR with/without laboratory blood gas analysis; 3) results were reported in sufficient detail to allow for a reconstruction of contingency tables of the original data (true-positive, true-negative, false-positive and false-negative results); and 4) studies conducted in human newborns. Studies were excluded if they did not meet the inclusion criteria above. Duplicated studies, reviews and conference abstracts were rejected. In cases of data duplication (i.e., the same data was published in two or more reports), only the most recent study with the largest and most recent data set was included.

\section{Data extraction and quality assessment}

Two authors (J. L and HR. M) independently carried out the data extraction, including study characteristics, study design, demographic characteristics, blinding parameters, time between CXR and LUS and quantitative data that allowed for the construction of a standard $2 \times 2$ table. The Quality Assessment of Diagnostic Accuracy Studies-2 (QUADAS-2) tool was also applied by two authors (J. L and HR. M) independently to evaluate the methodologic quality of the selected studies [21]. This instrument consists of four domains: patient selection, index test, reference standard and flow and timing. Each domain was assessed with respect to the risk of bias. Discrepancies were resolved by discussion and a consensus with a third reviewer (WK. Y). Predefined meta-regression (subgroup analyses) was performed to explore potential sources of heterogeneity.

\section{Statistical analysis}

Data analysis was conducted using STATA (Version 14.0, Stata Corp LP) with user-written "midas" programs. The bivariate mixed effects model was used to 


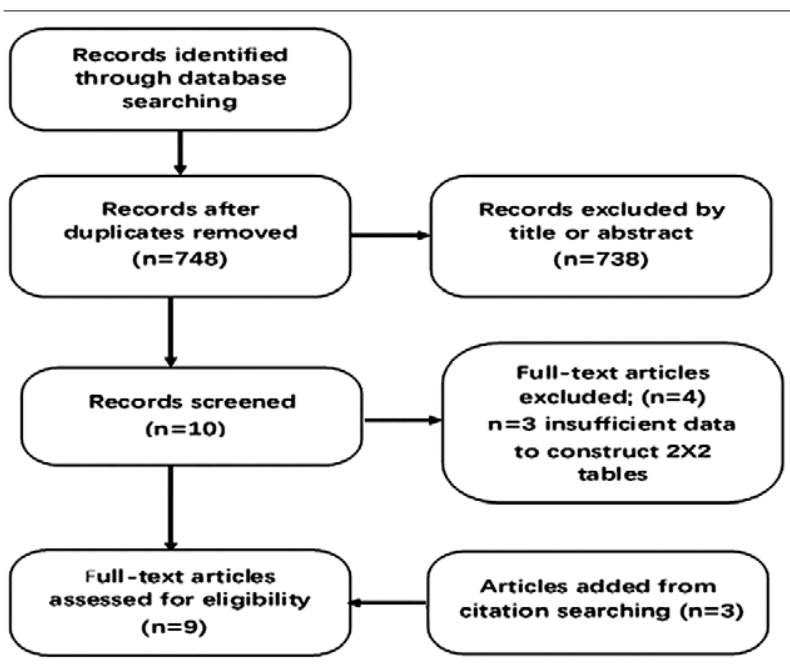

Fig 1. Preferred Reporting Items for Systematic Reviews and Meta-Analyses (PRISMA) flowchart of the search process

calculate pooled sensitivity and specificity with corresponding 95\% confidence intervals [22]. The diagnostic accuracy parameters mentioned above are shown graphically. Heterogeneity was analysed among studies using the $\mathrm{I}^{2}$ statistic and the $\mathrm{Q}$ test $[23,24]$. A summary receiver operator characteristic (SROC) curve was constructed to summarize the diagnostic value of LUS for NRDS. Publication bias was assessed using Deek's tests [25]. All statistical tests were two-sided with the type I error rate set as 0.05 . Thus, a p-value less than 0.05 was considered statistically significant.

\section{Results}

\section{Study flow and characteristics}

Our search retrieved 850 articles. After identifying 102 duplicated studies and excluding 738 studies based on predefined criteria, a total of 10 articles were selected for detailed review. In addition, 3 studies were manually added from the reference lists of the known reviews and the included primary studies and 4 studies were excluded due to various reasons summarized in figure 1, leaving a total of 9 studies for the final analysis. A total of 703 patients were included in this review. Six studies were prospective cohort studies $[9,12,16,17,26,27]$ and three studies were prospective case-control studies $[11,28,29]$. Detailed characteristics of the 9 studies are summarized in Table I and Table II.

\section{Primary outcome}

Nine studies assessed the diagnostic accuracy of LUS for NRDS in neonates and the assessment of methodologic quality using QUADAS-2 is illustrated in figure 2.

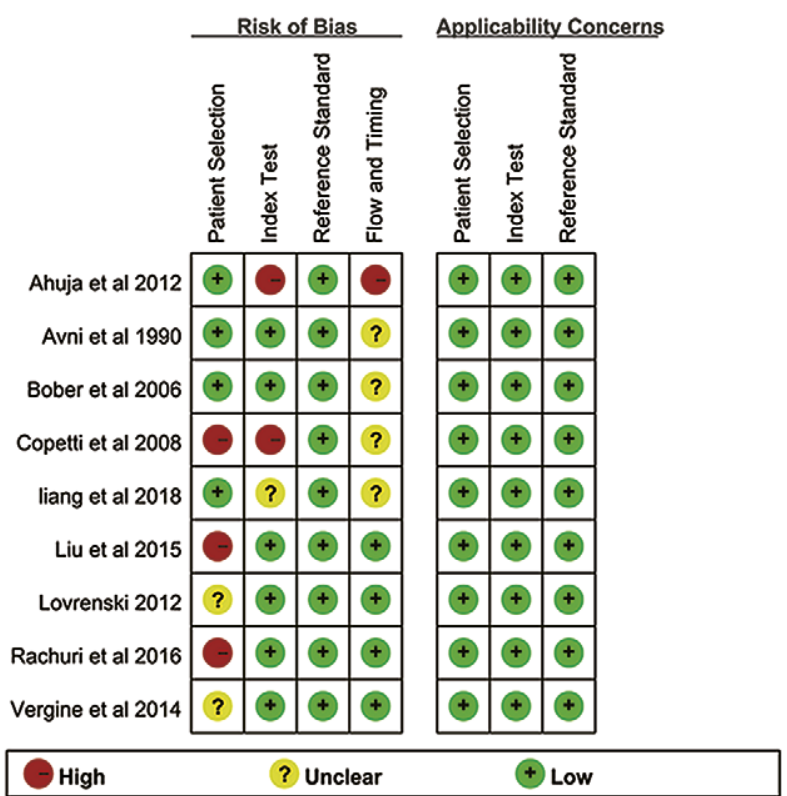

Fig 2. Methodological quality assessment according to QUADAS-2

Table I. Study characteristics

\begin{tabular}{lllllllll}
\hline Study & Origin & Study type & $\begin{array}{l}\text { Sample } \\
\text { size }\end{array}$ & $\begin{array}{l}\text { Gestational age } \\
(\text { mean } \pm \text { SD), weeks }\end{array}$ & $\begin{array}{l}\text { True } \\
\text { positive }\end{array}$ & $\begin{array}{l}\text { False } \\
\text { positive }\end{array}$ & $\begin{array}{l}\text { True } \\
\text { negative }\end{array}$ & $\begin{array}{l}\text { False } \\
\text { negative }\end{array}$ \\
\hline Ahuja, 2012 [12] & India & Prospective & 88 & $29^{+6} \pm 1.57$ & 32 & 6 & 44 & 6 \\
Avni, 1990 [9] & Belgium & Prospective & 40 & Unknown & 22 & 2 & 16 & 0 \\
Bober, 2006 [17] & Poland & Prospective & 131 & $32 \pm 4.4$ & 101 & 8 & 22 & 0 \\
Copetti, 2008 [11] & Italy & Case-control & 55 & $27.2 \pm 2.7$ & 40 & 0 & 15 & 0 \\
Liang, 2018 [26] & China & Prospective & 120 & $28.59 \pm 1.98$ & 29 & 4 & 82 & 5 \\
Liu, 2015 [28] & China & Case-control & 100 & $34.9 \pm 2.7$ & 50 & 0 & 50 & 0 \\
Lovrenski, 2012 [27] & Serbia & Prospective & 47 & $30.9 \pm 3.16$ & 43 & 0 & 2 & 2 \\
Rachuri, 2017 [29] & India & Case-control & 63 & $34.5 \pm 3.2$ & 29 & 1 & 33 & 0 \\
Vergine, 2014 [16] & Italy & Prospective & 59 & $33 \pm 4$ & 22 & 2 & 34 & 1 \\
\hline
\end{tabular}

$\mathrm{SD}$ - standard deviation 
Table II. Index test and reference characteristics

\begin{tabular}{|c|c|c|c|c|c|c|c|}
\hline Study & $\begin{array}{l}\text { Transducer } \\
\text { frequency } \\
(\mathrm{MHz})\end{array}$ & $\begin{array}{l}\text { LUS } \\
\text { operator }\end{array}$ & $\begin{array}{l}\text { QUDAS-2 } \\
\text { score } \\
(0-7)\end{array}$ & $\begin{array}{l}\text { Diagnostic } \\
\text { method }\end{array}$ & LUS technique & $\begin{array}{l}\text { Time between } \\
\text { CXR and } \\
\text { LUS }\end{array}$ & Blinding \\
\hline Ahuja, 2012 [12] & $5-12$ & Radiologist & 5 & $\begin{array}{l}\text { Gastric aspirate } \\
\text { test }+C D+C X R\end{array}$ & Transabdominal & $<20 \mathrm{~h}$ & No \\
\hline Avni, 1990 [9] & 5 & Unknown & 6 & $\mathrm{CD}+\mathrm{CXR}$ & Transabdominal & $<24 \mathrm{~h}$ & Yes \\
\hline Bober, 2006 [17] & 5 & Physician & 6 & $\begin{array}{l}\text { CRIB score+ } \\
\text { CXR+blood }\end{array}$ & Transabdominal & $<24 \mathrm{~h}$ & Yes \\
\hline Copetti, 2008 [11] & 10 & $\begin{array}{l}\text { Paediatrician+ } \\
\text { Cardiologist }\end{array}$ & 4 & $\mathrm{CD}+\mathrm{CXR}$ & Transthoracic & $<24 \mathrm{~h}$ & No \\
\hline Liang, 2018 [26] & $8-12$ & Unknown & 5 & CD+CXR+blood & Transthoracic & $<6 \mathrm{~h}$ & Unkonwn \\
\hline Liu, 2015 [28] & $>7.5$ & expert & 6 & CD+CXR+blood & Transthoracic & Immediate & Yes \\
\hline Lovrenski, 2012 [27] & 7.5 & $\begin{array}{l}\text { Paediatric } \\
\text { Radiologist }\end{array}$ & 6 & CD+CXR+blood & $\begin{array}{l}\text { Transthoracic }+ \\
\text { Transabdominal }\end{array}$ & $3.24-4.96 \mathrm{~h}$ & No \\
\hline Rachuri, 2017 [29] & $4-8 / 10-12$ & Radiologist & 6 & $\mathrm{CD}+\mathrm{CXR}$ & $\begin{array}{l}\text { Transthoracic }+ \\
\text { Transabdominal }\end{array}$ & $<4 \mathrm{~h}$ & Yes \\
\hline Vergine, 2014 [16] & $10-12$ & Neonatologist & 6 & $\mathrm{CD}+\mathrm{CXR}$ & Transthoracic & $<24 \mathrm{~h}$ & Yes \\
\hline
\end{tabular}

CD - Clinical diagnosis; CXR - Chest radiography; CRIB score - Clinical risk index for babies score; LUS - Lung ultrasound; QUDAS-2 - Quality assessment of diagnostic accuracy studies-2

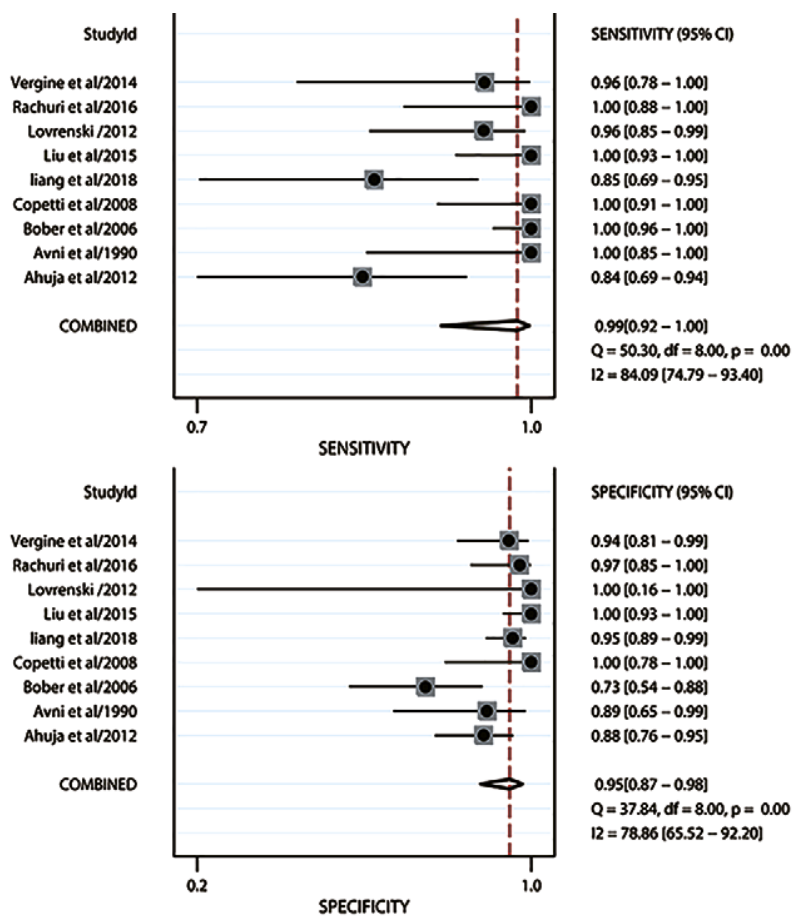

Fig 3. Forest plots of sensitivity and specificity

The pooled sensitivity and specificity of LUS for diagnosing NRDS were 0.99 (CI: 0.92-1.00) and 0.95 (CI: 0.87$0.98)$, respectively. The $\mathrm{Q}$ values were $50.30(\mathrm{p}=0.00)$ and $37.84(\mathrm{p}<0.00)$, indicating heterogeneity among the studies. The $\mathrm{I}^{2}$ statistic values were $84.09 \%$ and $78.86 \%$, respectively (fig 3). Since these values were $>50 \%$, this was considered to be significant heterogeneity based on the recommendations from the Cochrane handbook [30]. This supports the use of meta-regression (subgroup analyses) to explore meta-analysis results. Additionally, the AUROC was 0.99 (0.98-1.0) (fig 4). Publication bias was evaluated using Deek's funnel plot asymmetry test and was suggested to be not statistically significant $(\mathrm{p}=0.80$; fig 5). Consequently, we did not apply the trim and fill approach [31].

Meta-regression analysis of the six prospective cohort studies showed a pooled sensitivity of $96 \%$ (CI:

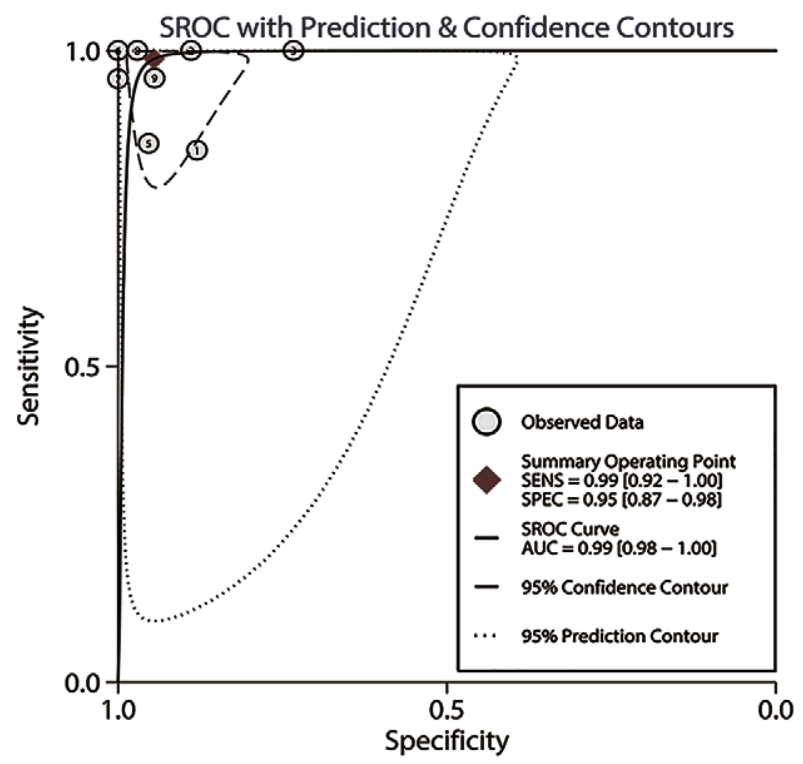

Fig 4. Summary receiver operating characteristic curves. 


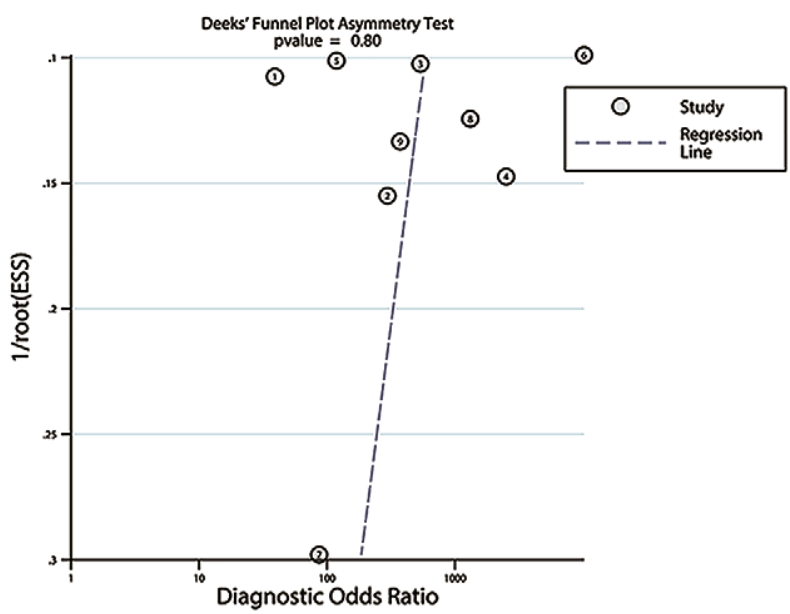

Fig 5. Summary receiver operating characteristic curves.

91-100\%) and specificity of $89 \%$ (CI: 84-95\%) and three prospective case-control studies showed a pooled sensitivity of $100 \%$ (CI: $100-100 \%$ ) and specificity of $99 \%$ (CI: $97-100 \%$ ). For the six studies that used transthoracic scanning, the LUS sensitivity was 99\% (CI: 96-100\%) and the specificity was $97 \%$.

In comparison, the pooled sensitivity of the three studies that used transabdominal scanning only was $99 \%$ (CI: $95-100 \%$ ) and the specificity was 83\% (CI: 76-91\%). In four studies with intervals between CXR and LUS less than 6 hours, the pooled sensitivity was 98\% (CI: 94$100 \%$ ) and the specificity was $98 \%$ (CI: $95-100 \%$ ). However, in five studies with intervals less than 24 hours, the pooled sensitivity was $99 \%$ (CI: $97-100 \%$ ) and the specificity was $89 \%$ (CI: 82-97\%). For sensitivity and specificity, the "type" of study was a statistically significant factor $(p<0.05)$ and the sensitivity of the "technology" in the studies was approximately significant $(\mathrm{p}=0.05)$. The detailed data are presented in figure 6 .

\section{Discussion}

In this study, we found that LUS was valuable for the detection of neonatal respiratory distress syndrome (NRDS). LUS showed high sensitivity (99\%) and specificity $(95 \%)$ in detecting NRDS. CXR is currently a common reference tool in the diagnosis of NRDS [5]. Vergine et al. demonstrated a sensitivity and specificity of $91 \%$ and $84 \%$ for CXR in diagnosing NRDS, respectively [16]. When using CXR to detect NRDS, Liang et al [26] showed that this method has a sensitivity of $88.23 \%$ and a specificity of $89.53 \%$. Copetti et al [11] described that the simultaneous presence of abnormalities in the pleural line, ultrasound aspect of "white lung" and the absence of "spared areas" has a sensitivity and specificity of $100 \%$.

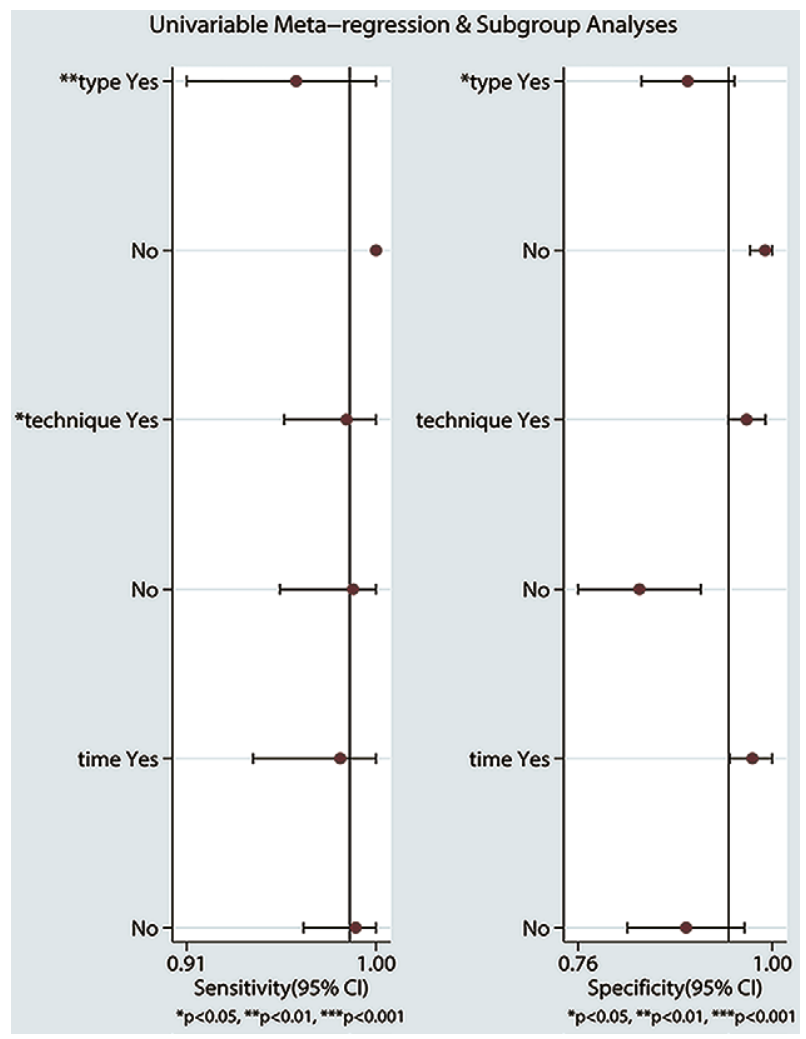

Fig 6. Meta-regression analysis of the meta-regression results

Based on these data, the accuracy of LUS was better than that of CXR. The pooled PLR and NLR were 18.1 (7.5, $43.4)$ and $0.01(0.00,0.09)$, respectively, which also indicated a high level of overall diagnostic accuracy.

Heterogeneity was found in this meta-analysis through the $\mathrm{I}^{2}$ statistic and the $\mathrm{Q}$ test $\left(\mathrm{I}^{2}>50, \mathrm{p}<0.01\right)$. Subgroup analysis revealed a significant difference between case-control studies and cohort studies. The reason for this is that enrolling participants with known disease and a control group without the condition may similarly exaggerate the diagnostic accuracy $[21,32,33]$. As such, we believed that a subgroup analysis of the six cohort studies would provide the most accurate reflection of test accuracy, although both subgroup analyses had high sensitivity and specificity.

Apart from the significant differences in study design in the subgroup analysis, there are several possible sources for this heterogeneity. First, studies that adopted the transthoracic technique as the detection method had a non-significantly higher diagnostic accuracy compared with studies that used the transabdominal approach as the detection method. Copetti et al [11] claimed that, in comparison with the transabdominal approach, the transthoracic technique could examine all the lung fields rather than only the base. This finding is in accordance 
with the findings of Hiles et al [34], suggesting that the transthoracic technique may be the better diagnostic approach for avoiding false-positive diagnoses and has the clinical advantage of reducing unnecessary additional testing and interventions. Second, the subgroup analysis also indicated that studies with a detection interval between CXR and LUS of less than 6 hours had a nonsignificantly higher specificity than those with a detection interval less than 24 hours. Only one study [28] in this meta-analysis performed LUS and CXR at the same time, which is ideal. The CXR and LUS features of RDS change following progressive pathology exacerbation and/or management of the disease. If a delay occurs or if treatment of NRDS begins between LUS and CXR, the improvement or deterioration of the condition may be misclassified. An inappropriate interval between the index test and the reference standard may be problematic for the diagnosis of acute diseases [21]. Vergine et al [16] declared that the sonographic appearance of RDS does not change immediately after surfactant administration. This may be explained by data obtained with an animal model that confirmed that there is no difference in fluid lung content among lungs treated or not treated with surfactants [35]. Oktem et al [36] demonstrated that LUS did not find any valuable changes or differences 2 hours after surfactant replacement therapy and that changes occurred 4 hours after surfactant therapy. However, the optimal test interval between LUS and CXR is still unknown. Third, three studies $[11,12,27]$ included in this review did not show "blinding" while performing LUS and one study had unknown blinding parameters [26]. Knowledge of the clinic and-radiologic data may overestimate the diagnostic accuracy of LUS [37]. Therefore, different LUS methods might contribute to the heterogeneity in the meta-analysis. Fourth, the choice of standard criteria for the diagnosis of NRDS may influence the diagnostic accuracy of LUS. If patients are diagnosed with a different reference standard, verification bias can occur [21]. Studies that adopted only "clinical diagnosis and CXR" as the standard criterion may show a lower diagnostic accuracy than those that used a combination of "clinical diagnosis, CXR and blood results (or gastric aspirate shake test)" as the standard criterion. More studies on the standard criteria would provide more details for the diagnosis of NRDS. Currently, the little information regarding the standard criteria for a reference test may overlook patients with NRDS, resulting in an underestimated diagnostic accuracy of LUS for NRDS.

CXR has potential harmful effects on infants. Infants are more sensitive to radiation than adults $[6,8]$. Hence, LUS has been recently recommended as a routine procedure for the diagnosis of different neonatal lung patholo- gies [38-45] in the neonatal intensive care unit (NICU). Oktem et al [36] and Brat et al [46] emphasized the importance of LUS in monitoring NRDS treatment. Repeated CXR for infants in a short time is unethical and harmful due to the increased exposure to ionizing radiation. Gargani et al [47] showed that sonographic abnormalities in the lung precede $\mathrm{PaO}_{2} / \mathrm{FiO}_{2}$ changes in an animal model. Therefore, LUS can be easily used for the early detection of patients with NRDS before clinical deterioration and improve the clinical progression and prognosis.

The mechanism of LUS for assessing lung pathologies is based on the artefact phenomenon. Techniques for detecting NRDS have been developed and include the transabdominal approach and the transthoracic approach, and it seems that the diagnostic accuracy of the latter method is better than that of the former. Copetti et al [48] demonstrated that the transabdominal approach could not evaluate the changes in the pleural line, which are essential for the diagnosis of NRDS. Liu et al [28] suggested that combined imaging findings of lung consolidation, pleural line abnormalities and white lung by the transthoracic method had a high diagnostic accuracy for NRDS with a sensitivity and specificity of $100 \%$. This might explain why Ahuja et al [12] found a lower diagnostic accuracy using transabdominal ultrasound for the diagnosis of NRDS. Their studies showed a sensitivity of $84.2 \%$ and specificity of $88 \%$.

Despite the favourable performance of LUS in the diagnosis of NRDS, we should be aware of several concerns. For example, subscapular or subclavicular consolidations or perihilar consolidations that do not reach the pleura cannot be evaluated by ultrasound imaging [49]. Copetti et al [11] maintained that air-leak syndrome (pneumomediastinum, interstitial emphysema, pneumopericardium) and complications of NRDS cannot be detected using LUS. Nonetheless, June et al [50] claimed that pneumomediastinum could be easily visualized and abnormal air accumulation could be localized by ultrasound.

The severity of NRDS is divided into 4 stages on the basis of CXR. These stages of severity correlate closely with the management of NRDS. Bober et al [17] indicated that the stages on ultrasound examination were well correlated to the radiographic criteria for stages. Liu et al [28] argued that LUS may not be proper for the diagnosis of grade I NRDS.

The interpretation of the basic LUS patterns is quite important for the diagnosis of NRDS. Tsou et al [51] indicated that the diagnostic accuracy of lung ultrasound is significantly affected by sonographer experience. This is similar to CXR since there are variations in intra-observer and inter-observer agreement among radiologists 
who use CXR for diagnosis [52]. However, Copetti et al claimed that inter- and intra-observer variability in the interpretation of imaging findings is a theoretical problem, not a real problem [11]. Bedetti et al [53] showed that ultrasound lung comets are equally reliable between experienced operators and new beginners.

This study has several limitations that merit consideration. First, only 9 studies were included in the review, which might not represent all the clinical features of NRDS. This may be because LUS is still a relatively new technique and further studies will be required to guarantee LUS as a helpful diagnostic method for NRDS. Second, there is significant heterogeneity in this meta-analysis. Hence, our findings should be interpreted after taking this factor into consideration and the diagnostic accuracy of LUS for NRDS may not be high enough. In addition to the possible reasons discussed above, heterogeneity may be affected by variations in gestational age and operators [51] and may also depend on the probe that is used. Future studies should explore ways to reduce these variations, ensure repeatability of these results and identify the optimal probe needed for this purpose [54].

\section{Conclusion}

Our findings show that LUS is an accurate diagnostic tool for NRDS, has the benefits of being a low-cost, nonionizing and repeatable method that can be performed at the bedside and has a comparable predictive power compared with CXR. Nevertheless, it is important to note that this conclusion is based on a small number of studies and the heterogeneity should be taken into account. Methodological stringency and multicenter studies are required to confirm this conclusion and further assess the performance of LUS in the diagnosis of NRDS.

\section{Conflict of interest: none}

Acknowledgment: This work was supported by the Social Development Projects, Beijing Chaoyang District Bureau of Science, Technology and Information (CYSF1922).

\section{References}

1. Warren JB, Anderson JM. Newborn respiratory disorders. Pediatr Rev 2010;31:487-495.

2. Stoll BJ, Hansen NI, Bell EF, et al. Neonatal outcomes of extremely preterm infants from the NICHD Neonatal Research Network. Pediatrics 2010;126:443456.

3. Anadkat JS, Kuzniewicz MW, Chaudhari BP, Cole FS, Hamvas A. Increased risk for respiratory distress among white, male, late preterm and term infants. J Perinatol 2012;32:780-785.
4. Development of audit measures and guidelines for good practice in the management of neonatal respiratory distress syndrome. Report of a Joint Working Group of the British Association of Perinatal Medicine and the Research Unit of the Royal College of Physicians. Arch Dis Child 1992;67(10 Spec No):1221-1227.

5. Sweet DG, Carnielli V, Greisen G, et al. European Consensus Guidelines on the Management of Respiratory Distress Syndrome - 2019 Update. Neonatology 2019;115:432-450.

6. Mazrani W, McHugh K, Marsden PJ. The radiation burden of radiological investigations. Arch Dis Child 2007;92:1127-1131.

7. Frush DP, Donnelly LF, Rosen NS. Computed tomography and radiation risks: what pediatric health care providers should know. Pediatrics 2003;112:951-957.

8. Hall EJ. Lessons we have learned from our children: cancer risks from diagnostic radiology. Pediatr Radiol 2002;32:700-706.

9. Avni EF, Braude P, Pardou A, Matos C. Hyaline membrane disease in the newborn: diagnosis by ultrasound. Pediatr Radiol 1990;20:143-146.

10. Avni EF, Cassart M, de Maertelaer V, Rypens F, Vermeylen D, Gevenois PA. Sonographic prediction of chronic lung disease in the premature undergoing mechanical ventilation. Pediatr Radiol 1996;26:463-469.

11. Copetti R, Cattarossi L, Macagno F, Violino M, Furlan R. Lung ultrasound in respiratory distress syndrome: a useful tool for early diagnosis. Neonatology 2008;94:52-59.

12. Ahuja CK, Saxena AK, Sodhi KS, Kumar P, Khandelwal $\mathrm{N}$. Role of transabdominal ultrasound of lung bases and follow-up in premature neonates with respiratory distress soon after birth. Indian J Radiol Imaging 2012;22:279-283.

13. Liu J, Cao HY, Wang HW, Kong XY. The role of lung ultrasound in diagnosis of respiratory distress syndrome in newborn infants. Iran J Pediatri 2014;24:147-154.

14. El-Malah HEDGM, Hany S, Mahmoud MK, Ali AM. Lung ultrasonography in evaluation of neonatal respiratory distress syndrome. Egypt J Radiol Nucl Med 2015;46:469474.

15. Rachuri H, Oleti TP, Murki S, Subramanian S, Nethagani J. Diagnostic Performance of Point of Care Ultrasonography in Identifying the Etiology of Respiratory Distress in Neonates. Indian J Pediatr 2017;84:267-270.

16. Vergine M, Copetti R, Brusa G, Cattarossi L. Lung ultrasound accuracy in respiratory distress syndrome and transient tachypnea of the newborn. Neonatology 2014;106:8793.

17. Bober K, Świetliński J. Diagnostic utility of ultrasonography for respiratory distress syndrome in neonates. Med Sci Monitor 2006; 12:CR440-CR446.

18. Whiting P, Westwood M, Burke M, Sterne J, Glanville J. Systematic reviews of test accuracy should search a range of databases to identify primary studies. J Clin Epidemiol 2008;61:357-364.

19. van Enst WA, Scholten RJ, Whiting P, Zwinderman AH, Hooft L. Meta-epidemiologic analysis indicates that MEDLINE searches are sufficient for diagnostic test accu- 
racy systematic reviews. J Clin Epidemiol 2014;67:11921199.

20. Beynon R, Leeflang MM, McDonald S, et al. Search strategies to identify diagnostic accuracy studies in MEDLINE and EMBASE. Cochrane Database Syst Rev 2013(9):Mr000022.

21. Whiting PF, Rutjes AW, Westwood ME, et al. QUADAS-2: a revised tool for the quality assessment of diagnostic accuracy studies. Ann Intern Med 2011;155:529-536.

22. Reitsma JB, Glas AS, Rutjes AW, Scholten RJ, Bossuyt PM, Zwinderman AH. Bivariate analysis of sensitivity and specificity produces informative summary measures in diagnostic reviews. J Clin Epidemiol 2005;58:982990.

23. Higgins JP, Thompson SG, Deeks JJ, Altman DG. Measuring inconsistency in meta-analyses. BMJ 2003;327:557560 .

24. Cohen JF, Chalumeau M, Cohen R, Korevaar DA, Khoshnood B, Bossuyt PM. Cochran's Q test was useful to assess heterogeneity in likelihood ratios in studies of diagnostic accuracy. J Clin Epidemiol 2015;68:299-306.

25. van Enst WA, Ochodo E, Scholten RJ, Hooft L, Leeflang MM. Investigation of publication bias in meta-analyses of diagnostic test accuracy: a meta-epidemiological study. BMC Med Res Methodol. 2014;14:70.

26. Liang H, Chen Z, Liao J, Yang H, Tan X. Diagnostic efficacy of lung ultrasound and X-ray for neonatal respiratory distress syndrome and transient tachypnea. Chinese Journal of Medical Imaging Technology 2018;34:683687.

27. Lovrenski J. Lung ultrasonography of pulmonary complications in preterm infants with respiratory distress syndrome. Ups J Med Sci 2012;117:10-17.

28. Liu J, Cao HY, Wang HW, Kong XY. The role of lung ultrasound in diagnosis of respiratory distress syndrome in newborn infants. Iran J Pediatr 2014;24:147-154.

29. Rachuri H, Oleti TP, Murki S, Subramanian S, Nethagani J. Diagnostic Performance of Point of Care Ultrasonography in Identifying the Etiology of Respiratory Distress in Neonates. Indian J Pediatr 2017;84:267-270.

30. Higgins JPT, Thomas J, Chandler J, Cumpston M, Li T, Page MJ, Welch VA (editors). Cochrane Handbook for Systematic Reviews of Interventions version 6.0 (updated July 2019). Cochrane, 2019 Available from: wwwtrainingcochraneorg/handbook.

31. Duval S, Tweedie R. Trim and fill: A simple funnel-plotbased method of testing and adjusting for publication bias in meta-analysis. Biometrics 2000;56:455-463.

32. Lijmer JG, Mol BW, Heisterkamp S, et al. Empirical evidence of design-related bias in studies of diagnostic tests. JAMA 1999;282:1061-1066.

33. Whiting PF, Rutjes AW, Westwood ME, Mallett S; QUADAS-2 Steering Group. A systematic review classifies sources of bias and variation in diagnostic test accuracy studies. J Clin Epidemiol 2013;66:1093-1104.

34. Hiles M, Culpan AM, Watts C, Munyombwe T, Wolstenhulme S. Neonatal respiratory distress syndrome: Chest
X-ray or lung ultrasound? A systematic review. Ultrasound 2017;25:80-91.

35. Cattarossi L, Copetti R, Poskurica B, Miserocchi G. Surfactant administration for neonatal respiratory distress does not improve lung interstitial fluid clearance: echographic and experimental evidence. J Perinat Med 2010;38:557563.

36. Oktem A, Yigit S, Oguz B, Celik T, Haliloglu M, Yurdakok M. Accuracy of lung ultrasonography in the diagnosis of respiratory distress syndrome in newborns. J Matern Fetal Neonatal Med 2019. doi:10.1080/14767058.2019.1605350.

37. Whiting P, Rutjes AW, Reitsma JB, Glas AS, Bossuyt PM, Kleijnen J. Sources of variation and bias in studies of diagnostic accuracy: a systematic review. Ann Intern Med 2004;140:189-202.

38. Liu J, Liu Y, Wang HW, et al. [Lung ultrasound for diagnosis of neonatal atelectasis]. Zhonghua Er Ke Za Zhi 2013;51:644-648.

39. Liu J, Chen XX, Li XW, Chen SW, Wang Y, Fu W. Lung Ultrasonography to Diagnose Transient Tachypnea of the Newborn. Chest 2016;149:1269-1275.

40. Liu J, Cao HY, Fu W. Lung ultrasonography to diagnose meconium aspiration syndrome of the newborn. J Int Med Res 2016;44:1534-1542.

41. Desjardins MP, Weerdenburg KD, Fischer JW. Emergency Point-of-Care Ultrasound Diagnosis of Diaphragmatic Hernia in the Pediatric Emergency Department. Pediatr Emerg Care 2016;32:685-687.

42. Liu J, Chi JH, Ren XL, et al. Lung ultrasonography to diagnose pneumothorax of the newborn. Am J Emerg Med 2017;35:1298-1302.

43. Liu J, Liu F, Liu Y, Wang HW, Feng ZC. Lung ultrasonography for the diagnosis of severe neonatal pneumonia. Chest 2014; 146:383-388.

44. Scholl JE, Yanowitz TD. Pulmonary hemorrhage in very low birth weight infants: a case-control analysis. J Pediatr 2015;166:1083-1084.

45. Del Rey Hurtado de Mendoza B, Sanchez-de-Toledo J, Bobillo Perez S, Girona M, Balaguer Gargallo M, RodriguezFanjul J. Lung Ultrasound to Assess the Etiology of Persistent Pulmonary Hypertension of the Newborn (LUPPHYN Study): A Pilot Study. Neonatology 2019;116:140-146.

46. Brat R, Yousef N, Klifa R, Reynaud S, Shankar Aguilera S, De Luca D. Lung Ultrasonography Score to Evaluate Oxygenation and Surfactant Need in Neonates Treated With Continuous Positive Airway Pressure. JAMA Pediatr 2015;169:e151797.

47. Gargani L, Lionetti V, Di Cristofano C, Bevilacqua G, Recchia FA, Picano E. Early detection of acute lung injury uncoupled to hypoxemia in pigs using ultrasound lung comets. Critical Care Med 2007;35:2769-2774.

48. Copetti R, Cattarossi L. The 'double lung point': an ultrasound sign diagnostic of transient tachypnea of the newborn. Neonatology 2007;91:203-209.

49. Najgrodzka P, Buda N, Zamojska A, Marciniewicz E, Lewandowicz-Uszynska A. Lung Ultrasonography in the Diagnosis of Pneumonia in Children-A Metaanalysis 
and a Review of Pediatric Lung Imaging. Ultrasound Q 2019;35:157-163.

50. Jung AY, Yang I, Go HS, et al. Imaging neonatal spontaneous pneumomediastinum using ultrasound. J Med Ultrason 2014;41:45-49.

51. Tsou PY, Chen KP, Wang YH, et al. Diagnostic Accuracy of Lung Ultrasound Performed by Novice Versus Advanced Sonographers for Pneumonia in Children: A Systematic Review and Meta-analysis. Acad Emerg Med 2019;26:1074-1088.
52. Copetti R, Cattarossi L. Ultrasound diagnosis of pneumonia in children. Radiol Med 2008;113:190-198.

53. Bedetti G, Gargani L, Corbisiero A, Frassi F, Poggianti E, Mottola G. Evaluation of ultrasound lung comets by handheld echocardiography. Cardiovasc Ultrasound 2006;4:34.

54. Raimondi F, Yousef N, Migliaro F, Capasso L, De Luca D. Point-of-care lung ultrasound in neonatology: classification into descriptive and functional applications. Pediatr Res 2018 Jul 20. doi:10.1038/s41390-018-0114-9. 